\title{
Efectividad del programa piloto de intervención escolar Tejedores de Resiliencia en niños
}

Volumen 6 N.o 48 enero-junio de 2020

ISSN: 0122-4328

ISSN-E: 2619-6069

pp. 131-147

Effectiveness

of Tejedores de

Resiliencia school

intervention pilot

program in children
Eficácia do programa piloto de intervenção escolar Tejedores de Resiliencia em crianças

María Alejandra Ávila Moreno*

Neila Stella Díaz Bahamón**

Fecha de recepción: 23-06-19

Fecha de aprobación: 22-02-20

PARA CITAR ESTE ARTÍCULO

Ávila Moreno, M. y Díaz Bahamón, N. (2020). Efectividad del programa piloto de intervención escolar Tejedores de Resiliencia en niños. Nodos y Nudos, 6(48). https://doi.org/ 10.17227/nyn.vol6.num48-10213

* Universidad de la Sabana. mariaavimo@unisabana.edu.co

* Universidad de la Sabana.neila.diaz@unisabana.edu.co 


\section{RESUMEN}

Estudios recientes demuestran que la escuela es el lugar clave para desarrollar resiliencia en niños que han estado expuestos a condiciones de vulnerabilidad social. Esto a partir de programas de intervención que incorporen tanto la reducción de los factores de riesgo, como el fortalecimiento de los factores de protección, en todos los sistemas de los cuales hacen parte. Siguiendo esta línea, el objetivo de este estudio de carácter exploratorio, preexperimental, bajo el diseño pretest -intervención- postest, con un solo grupo, fue evaluar la efectividad del programa piloto de intervención escolar Tejedores de Resiliencia en los niveles de resiliencia de niños en condición de vulnerabilidad social. Para las mediciones, se utilizó el instrumento Inventario de Factores Personales de Resiliencia, el cual evalúa el constructo resiliencia general a través de cinco factores personales: autoestima, empatía, autonomía, humor y creatividad. En la implementación del programa participaron 115 niños entre 7 y 10 años $(=8,22 \mathrm{~s}=, 646)$, estudiantes de grado tercero de primaria de una Institución Educativa Distrital en la ciudad de Bogotá, junto con sus padres o acudientes, las docentes directoras de curso y la comunidad circundante a la escuela, durante ocho meses. El efecto del programa se evaluó mediante diferencia de medias. Los resultados sugieren que la intervención aumentó los niveles de resiliencia general, así como los factores autoestima y humor en los estudiantes. No hubo cambios significativos en los factores de empatía, autonomía y creatividad.

Palabras clave: resiliencia; programa de intervención; modelo ecológico; factores de protección; factores de riesgo

\section{ABSTRACT}

Recent studies demonstrate that school is the perfect place to develop resilience in children who have been exposed to conditions of social vulnerability. This is based on intervention programs that incorporate both the reduction of risk factors and the strength of protective factors in all the systems they take part in. According to this, the aim of this exploratory pre-experimental study, under the pretest -intervention- posttest design, and with a single group, was to evaluate the effectiveness of the pilot program of school intervention Tejedores de Resiliencia, in the resilience levels of children with a condition of social vulnerability. The instrument used for the measurement was the Personal Resilience Factors Inventory which evaluates the general resilience construct through five personal factors: self-esteem, empathy, autonomy, emotional state, and creativity. 115 children between 7 and 10 years old $(=8,22 \mathrm{~s}=, 646)$, third-grade students of a Public School in the city of Bogota, participated in the implementation of the program together with their parents or legal guardians, teachers, and the community surrounding the school, for eight months. The difference of means was used to evaluate the effect of the program. The results suggest that this intervention increased the levels of general resilience, as well as the factors of self-stem and emotional state in the students. There were no significant changes in empathy, autonomy, and creativity factors.

Keywords: resilience; intervention program; ecological model; protection factors; risk factors

\section{RESUMO}

Estudos recentes mostram que a escola é o local chave para desenvolver resiliência em crianças que foram expostas a condições de vulnerabilidade social. Baseia-se em programas de intervenção que incorporam quer a redução dos fatores de risco, quer o reforço dos fatores de proteção, em todos os sistemas de que fazem parte. Seguindo essa linha, o objetivo deste estudo exploratório, pré-experimental, sob o delineamento pré-teste-intervenção-pós-teste, com um único grupo, foi avaliar a eficácia do programa piloto de intervenção escolar de Tejedores de Resiliencia nos niveis de resiliência de crianças em situação de vulnerabilidade social. Para as medidas, foi utilizado o instrumento Inventário de Fatores de Resiliência Pessoal, que avalia o construto geral da resiliência por meio de cinco fatores pessoais: autoestima, empatia, autonomia, humor e criatividade. Participaram da implementação do programa 115 crianças entre 7 e $10 \operatorname{anos}(=8,22 \mathrm{~s}=0,646)$, alunos da terceira série de uma Instituição Educacional Distrital da cidade de Bogotá, juntamente com seus pais ou responsáveis, os diretores de cursos e a comunidade do entorno da escola, por oito meses. 0 efeito do programa foi avaliado por meio da diferença das meias. Os resultados sugerem que a intervenção aumentou os niveis de resiliência geral, bem como os fatores de autoestima e humor nos alunos. Não houve mudanças significativas nos fatores de empatia, autonomia e criatividade.

Palavras-chave: resiliência; programa de intervenção; modelo ecológico; fatores de proteção; fatores de risco 


\section{Introducción}

El ser humano está, en mayor o menor medida, en un estado de vulnerabilidad constante, dado que son muchos los peligros y dificultades presentes en cada etapa de su ciclo vital, en particular en la niñez. Los niños se encuentran en un periodo de construcción y crecimiento tanto físico como mental. Su falta de experiencia y conocimiento los hace especialmente indefensos ante cualquier riesgo. Por ello, es relevante establecer al menos un vínculo afectivo y constructivo estrecho con un referente, como la familia o la escuela, que garantice protección, cuidados especiales y las condiciones para convertirse en miembros autónomos de la sociedad (Casillas, 2008; Lázaro, 2009; Obando et ál., 2015). Desafortunadamente, muchos niños no cuentan con este referente que promueva su desarrollo armónico e integral, o dicho referente no tiene las condiciones para satisfacer sus necesidades y no trasciende en su labor de acompañamiento.

Tal es el caso de la población infantil en edad escolar con la cual se llevó a cabo este estudio, dada su exposición directa a situaciones de pobreza (carencia efectiva y actual, debida a la precariedad en la inserción laboral de sus padres o acudientes), fragilidad en los vínculos relacionales, exposición al consumo de sustancias psicoactivas en su entorno inmediato, y a bandas delincuenciales dedicadas al microtráfico. En algunos participantes, identificados de forma concreta, se suman situaciones de abandono o negligencia parental, y maltrato físico o psicológico. Dichos riesgos se engloban desde esta perspectiva teórica en el concepto de vulnerabilidad social (Perona y Rocchi, 2001), además se originan y gestan fundamentalmente en el contexto familiar. De alli se desprende la necesidad de consolidar el ámbito escolar como el referente de protección y cuidado, el cual posibilita la intervención que aqui se propone.

Richaud (2013) señala que: "la vulnerabilidad social es una combinación de eventos, procesos 0 rasgos que constituyen adversidades o riesgos potenciales en el ejercicio de diferentes tipos de derechos de ciudadanía o el logro de los objetivos de proyectos comunitarios, familiares e individuales" ( $p$. 751). La vulnerabilidad social surge de la interacción entre factores de riesgo tanto internos como externos de la unidad de análisis, deteriorando su bienestar de diversas formas y con diferentes intensidades. Comprende tanto la exposición al riesgo como la disposición para enfrentarlo, lo que implica una adaptación activa que lleva a no sucumbir ante la adversidad y, por el contrario, realizar reestructuraciones internas que revaloricen el riesgo y lo conviertan en una oportunidad de crecimiento (Villalta y Saavedra, 2012). Esta interacción es lo que los autores han denominado resiliencia.

El término resiliencia procede del latín resilio, que significa volver atrás, volver de un salto, resaltar, rebotar, volver al estado original, recuperar la forma original. El prefijo "re" indica repetición, reanudación y describe la cualidad de materiales que resisten los impactos y recuperan su forma original cuando son forzados a deformarse. Es claramente un concepto nacido de la física y utilizado en la termodinámica y en la ingeniería civil, que luego fue adaptado a las ciencias sociales (Becoña, 2006; Casillas, 2008; Palacios y Monroy, 2011).

En el campo de la psicología no hay un completo acuerdo sobre la definición de resiliencia, ya que existen diferentes modelos que la conceptualizan. En la literatura actual es común encontrar dos puntos de vista: resiliencia como capacidad o como proceso. En el primer caso, los autores refieren que es la capacidad propiamente humana para hacer frente a la tragedia, el trauma, la adversidad y el estrés significativo, además superarlo, aprender de ello e inclusive ser transformado. Esto implica el empleo de competencias afectivas, sociales y de comunicación que pueden variar a lo largo del tiempo y las etapas de la vida (Becoña, 2006; Casillas, 2008; Richaud, 2013; Ruiz, 2012).

En el segundo caso, se entiende como un proceso dinámico que cualquier individuo puede tener o implementar, y se presenta en diferentes grados y momentos. Abarca adaptación positiva en el contexto de la adversidad significativa, dando lugar a resultados funcionales en el desarrollo. Este proceso implica la interacción entre un rango de factores de riesgo y de protección, presentes desde el individuo hasta el ámbito social, que posibilitan superar el trauma y 
la adversidad de forma constructiva (Cabrera, Aya y Guevara, 2014; González-Arratia y Valdez, 2013; Luthar et ál. , 2000; Morelato, 2011; Palacios y Monroy, 2011; Rodríguez et ál., 2012; Rutter, 2012; Windle et ál., 2011).

Tras considerar estas dos posturas y para los fines pertinentes, este estudio comprende la resiliencia como un proceso que involucra experiencias y trayectorias individuales en constante interacción con el entorno, las cuales varían en el curso de la vida. Y no como una capacidad, característica o rasgo presente en los individuos de forma innata, dado que esta última concepción deja de lado los factores contextuales, las influencias ambientales y sociales.

Con el fin de entender la influencia dinámica de múltiples factores en el desarrollo humano y las interacciones mutuas entre el individuo y su medio ambiente, el modelo ecológico propuesto por Bronfenbrenner (1993/1994), compuesto de cinco sistemas interrelacionados y socialmente organizados, resulta eficiente para explicar e intervenir la resiliencia (Morelato, 2011). En el primer sistema, denominado microsistema, se identifican los roles del individuo en sus entornos inmediatos como lo son la familia, la escuela y el grupo de pares. El segundo sistema, el mesosistema, comprende los enlaces entre dos o más marcos: incluye vínculos entre familia y escuela, o familia y grupo de amigos. En el tercero, exosistema, se reconocen los entornos entre los cuales el individuo no está como participante activo, pero cuyos eventos influyen en su desarrollo, por ejemplo el espacio laboral de los padres, o los vínculos entre familia y comunidad. En el cuarto sistema, o macrosistema, se reconocen las variables sociales para una cultura particular, lo cual incluye creencias, valores, sistemas políticos y económicos, que el individuo internaliza de forma activa o pasiva e influencian sus comportamientos. En el último sistema, Bronfenbrenner (1993/1994) enuncia el cronosistema, el cual se relaciona con los acontecimientos históricos de determinada época y el cambio o consistencia en el tiempo del individuo y los entornos en los que vive.

Belsky (1980) adiciona a la estructura anterior un contexto también importante, denominado ontosistema. Dicho sistema se relaciona con las características internas del individuo y su historia personal. Morelato (2011) señala que el ontosistema está constituido por factores biológicos, psicológicos y neuropsicológicos. Menciona que integrar conceptos vinculados a la resiliencia a través de este modelo ecológico de seis sistemas permite una mejor comprensión del constructo como proceso dinámico. Además, los factores involucrados en el desarrollo de determinado individuo pertenecen a todos los sistemas mencionados e interactúan recíprocamente, dando lugar a un mecanismo de resiliencia o de vulnerabilidad.

La investigación de resiliencia como proceso ha delineado tres niveles principales para su estudio e intervención: 1) atributos de los propios individuos, 2) aspectos de sus familias, y 3 ) características de sus entornos sociales más amplios, esto es el nivel individual, el familiar y el social (Luthar et ál., 2000). Identificar estos tres niveles es clave para definir en cada uno cómo actúan e interactúan los factores que promueven la resiliencia, denominados de protección, y los factores que limitan su desarrollo, conocidos como de riesgo. Tan importantes son los unos como los otros, los de protección para potenciar e incrementar y los de riesgo para disminuir y contrarrestar (Becoña, 2006).

El reconocimiento de estos factores es esencial para diseñar estrategias de prevención e intervención apropiadas para las personas que enfrentan adversidades, en este caso niños que han estado expuestos a condiciones de vulnerabilidad social. Lynch et ál. (2004) señalan que, las intervenciones basadas en un marco de resiliencia que incorporen tanto la reducción del riesgo como el fortalecimiento de los factores protectores afectan de forma positiva y fomentan mejores resultados tanto en los niños vulnerables como en aquellos que no han vivenciado adversidad significativa. Masten (2001) ha argumentado que la resiliencia es ordinaria, más que extraordinaria, y por ello puede implementarse en cualquier individuo.

Entender la resiliencia como un proceso permite, además de reconocer su sentido dinámico, intervenir en ella afectando los tres niveles mencionados anteriormente $y_{1}$ de igual manera, los factores protectores y de riesgo presentes en cada uno. Así, por ejemplo, a nivel individual en los niños, uno de los factores 
protectores que contribuye a desarrollar la resiliencia es el tener expectativas realistas de lo que cada uno puede lograr de acuerdo a sus talentos, capacidades y recursos propios (Fiorentino, 2008). En el nivel familiar, las prácticas de crianza basadas en el reconocimiento positivo de las capacidades de los niños y el diálogo abierto que permita la adecuada expresión emocional funcionan como significativos factores protectores (Rodríguez et ál., 2012). Y a nivel social, son la escuela y el vecindario las fuentes generadoras de factores protectores en los niños. La unión vecinal fomenta el sentimiento de pertenencia, y genera conexiones y redes de apoyo que atenúan el impacto del riesgo (Morelato, 2011).

Los estudios demuestran que la escuela, como contexto cultural privilegiado para el aprendizaje de la vida y el segundo entorno natural del niño después de su familia, es el lugar clave para desarrollar resiliencia (Acevedo y Mondragón, 2005; Becoña, 2006; Casillas, 2008; Coholic, Eys y Lougheed, 2012; Lynch et al., 2004; Richaud, 2013; Rodríguez et ál., 2012). Es un factor protector en sí mismo dado que, en muchos de los casos, la vulnerabilidad proviene de los propios hogares y son las familias quienes la generan o permiten que suceda. Es así como la escuela se convierte en el escenario de revalorización después de la tragedia y tiene el papel trascendente de una "segunda casa", en la cual los niños son capaces de encontrar la seguridad negada en su ámbito familiar. Ellos pasan una considerable cantidad de tiempo alli y la adquisición de conocimientos va más allá de lo expuesto en el plan de estudios (Richaud, 2013). Hoy más que nunca la escuela es la institución social donde confluyen todos los saberes, conocimientos, roles, relaciones humanas y relaciones de poder. Es el lugar donde los niños aprenden diferentes formas de acercarse a la vida y a actuar en consecuencia (Casillas, 2008), formándose no solo a través de lecciones académicas, las cuales en apariencia son la razón más importante de su estadía, sino de lecciones que permearán su ser en lo más profundo y sentarán las bases de su construcción personal y social.

Acevedo y Mondragón (2005) señalan que fuera de la familia el adulto que mayor influencia positiva puede tener sobre un niño es su maestro favorito. Es un modelo y a él puede dirigirse en los momentos de crisis. La importancia del apoyo externo de un adulto ha sido documentada extensamente en la literatura de resiliencia infantil. Los maestros y las escuelas tienen el poder de transformar vidas, usan las fortalezas de sus estudiantes, sus intereses, sus metas y sueños como punto de comienzo para su aprendizaje. En el diálogo permanente entre educación y resiliencia se encuentra una posible alternativa para una formación social, ciudadana y humana (Campo et ál., 2012).

En consecuencia, se justifica la necesidad de diseñar, desarrollar, documentar y evaluar programas de intervención en la escuela que promuevan y favorezcan la resiliencia en los niños. Los salones de clase son bastante apropiados para su implementación porque el entorno estructurado, y de apoyo en sí mismo, constituye también un factor protector. Son muchas las experiencias significativas que soportan la importancia que tienen estos programas y que al evaluar su impacto demuestran cambios significativos en los niveles de resiliencia de los participantes.

Tal es el caso del programa Al's Pals: Kids Making Healthy Choices (Lynch et ál., 2004), el cual se constituyó como una iniciativa dirigida a niños de primera infancia y se realizó en diferentes ubicaciones geográficas de Estados Unidos, desde 1993 hasta el 2000. Su núcleo central fue la aplicación sistemática de la investigación en resiliencia, involucrando a estudiantes, maestros y padres de familia. La evaluación de los datos recogidos sobre los resultados de los niños indican consistentemente que la intervención es efectiva para fortalecer las habilidades relacionadas con el desarrollo de la resiliencia. La metodología de evaluación evolucionó de pre-experimental a diseño experimental y abarcó repeticiones de programas múltiples.

Una segunda experiencia significativa llevada a cabo durante el 2004 en la ciudad de Cali, Colombia, fue el Programa Piloto de Construcción de Ambientes Educativos Resilientes (Acevedo y Mondragón, 2005). Esta iniciativa acentuó su trabajo con docentes de primaria de un colegio, con los propósitos de capacitarlos en el campo de la resiliencia educativa; desarrollar una perspectiva para el trabajo desde la resiliencia con estudiantes afectados por el conflicto armado; diseñar, adaptar, construir y reconstruir 
materiales para ser aplicados en las escuelas; y recuperar experiencias desde la práctica pedagógica de los mismos docentes. Después de la sistematización de la experiencia, se evidenció que los docentes, usando las herramientas adquiridas en la capacitación, empezaron a elaborar sus propios materiales de trabajo y estrategias de construcción de resiliencia, apuntando a las necesidades de sus estudiantes en el aula de clase. Además, los resultados de las actividades que los docentes propusieron en su colegio para el año siguiente apuntaron al despliegue de la competencia social, sentido de identidad, autonomía y solución de problemas en los estudiantes.

Un tercer ejemplo es el programa Descubriendo la Vida (Reyes, 2007), implementado con estudiantes de 10 y 11 años de grado quinto de primaria en un colegio del distrito de San Luis, Lima (Perú). Su objetivo general fue identificar y ejecutar acciones que les permitieran a los niños fortalecer e incrementar su capacidad resiliente. Los objetivos específicos fueron identificar y ejecutar acciones para incrementar los factores personales de la resiliencia: autoestima, empatía, autonomía, humor y creatividad. Dichos objetivos fueron organizados a través de 5 módulos, que comprendieron 17 sesiones en total (una sesión introductoria, una sesión de despedida y tres sesiones por cada módulo), tres veces a la semana y por un espacio de hora y media por sesión. Se llegó a la conclusión de que el programa, en términos generales de resiliencia, sí produce efectos positivos, pero en cuanto a los factores no produce el mismo resultado, excepto en el caso de la empatía. Estos resultados se obtuvieron mediante una investigación exploratoria, cuasiexperimental bajo el diseño pretest - postest con un solo grupo.

Por último, se puede señalar la experiencia obtenida por Coholic et ál. (2012), a partir de la implementación del programa Holistic Arts-Based Group Program (HAP) para el desarrollo de la resiliencia y la autoestima en niños y jóvenes necesitados de Canadá. Su objetivo fue enseñar a los participantes cómo entender sus sentimientos y revelar sus fortalezas. Se evaluó la efectividad del programa usando grupos de comparación, control y medidas estandarizadas. Asimismo, se basó en investigaciones cualitativas anteriores con el HAP que demostraron su idoneidad, factibilidad y beneficios para la resiliencia y la autoestima, en un periodo previo de 3 años. Se encontró evidencia de que el HAP fue beneficioso para los niños; ellos mismos reportaron una menor reactividad emocional (una medida de resiliencia) después de la intervención. No se observaron cambios en las percepciones de autoestima. Se destacó que los métodos basados en las artes y los oficios pueden involucrar a los niños y jóvenes de una manera creativa, significativa y relevante, en un proceso que es agradable y centrado en sus fortalezas.

Siguiendo esta línea, el objetivo de esta investigación fue evaluar la efectividad del programa piloto de intervención escolar Tejedores de Resiliencia en los niveles de resiliencia de niños en condición de vulnerabilidad social. Este programa propone un modelo de trabajo lúdico-pedagógico por medio del cual se aumente la resiliencia de los niños participantes, fortaleciendo los factores protectores y disminuyendo los factores de riesgo, presentes a nivel individual, familiar y social.

\section{Método}

\section{Diseño}

Estudio de carácter exploratorio, preexperimental, bajo el diseño pretest -intervención- postest, con un solo grupo.

\section{Participantes}

Se utilizó una muestra de tipo no probabilistico ya que la elección del grupo participante fue intencional. Estuvo compuesto por 115 estudiantes que cursaban grado tercero de primaria en una Institución Educativa Distrital en la ciudad de Bogotá, 53 niñas (46,1\%) y 62 niños (53,9\%), entre las edades de 7 y 10 años ( $\bar{x}=$ $8,22 \mathrm{~s}=, 646)$. Los participantes se educaban y vivian en condiciones de vulnerabilidad social. Pertenecian a estrato socioeconómico 1 (bajo) y experimentaban situaciones de pobreza (carencia efectiva y actual, debida a precariedad en la inserción laboral de sus padres o acudientes), fragilidad en los vínculos relacionales, exposición al consumo de sustancias psicoactivas en su entorno inmediato, y a bandas delincuenciales dedicadas al microtráfico. En algunos participantes específicamente identificados, se suman situaciones de abandono o negligencia parental, y maltrato físico o psicológico. 
Además de los estudiantes, participaron sus padres o acudientes, las tres docentes directoras de curso y la comunidad circundante a la escuela.

\section{Instrumento}

Las mediciones pretest - postest se realizaron con el instrumento Inventario de Factores Personales de Resiliencia, diseñado por Salgado (2005a), el cual arroja un puntaje total compuesto de resiliencia general a partir de la sumatoria de los 5 factores resilientes identificados: autoestima, empatía, autonomía, humor y creatividad. El puntaje total compuesto de resiliencia general ofrece categorias interpretativas que a su vez expresan niveles de resiliencia: 1) muy bajo, 2) bajo, 3) promedio, 4) alto y 5) muy alto. Además, el instrumento permite reconocer el comportamiento de los 5 factores resilientes y su contribución a la resiliencia general, por medio de las categorías: 1) bajo, 2) medio y 3) alto. Se encuentra dirigido a población infantil, con edades comprendidas entre 7 y 12 años.

\section{Procedimiento}

Pretest:

La aplicación del instrumento (marzo de 2017) fue realizada por la investigadora principal (orientadora escolar de la institución) en los respectivos salones de clase de los estudiantes de manera grupal y en los horarios académicos acostumbrados de su jornada escolar. Esto previa consecución de los consentimientos informados de los padres de familia, los asentimientos informados de los niños, la autorización del coordinador general de la institución educativa y de las 3 docentes directoras de curso. El tiempo aproximado de aplicación fue de 45 minutos. En el caso de 6 niños identificados con dificultades cognitivas, de aprendizaje o de lecto-escritura, el instrumento se administró de manera individual en los momentos de la jornada escolar que las directoras de curso permitían, en un tiempo aproximado de 30 minutos. A todos los participantes se les explicó el objetivo de la investigación y su participación fue voluntaria, anónima y confidencial teniendo en cuenta aspectos éticos de la investigación, sin que se observaran abstenciones.
Intervención:

El programa piloto de intervención escolar Tejedores de Resiliencia fue implementado por la investigadora principal con los estudiantes (nivel individual), sus padres o acudientes (nivel familiar), las docentes directoras de curso y la comunidad circundante (nivel social), durante un año escolar de 8 meses (marzo noviembre de 2017). Este programa reconoce los tres niveles principales de intervención que ha propuesto la investigación de resiliencia como proceso (Luthar et al., 2000) y corresponde también al modelo ecológico de Bronfenbrenner (1993/1994) y Belsky (1980), al intervenir de modo directo desde el ontosistema hasta el exosistema de los niños participantes y afectar los factores de riesgo y de protección presentes en cada sistema. Además, sigue los lineamientos de algunos programas de intervención anteriores que han demostrado ser empíricamente efectivos (Acevedo y Mondragón, 2005; Coholic et al., 2012; Lynch et ál., 2004; Reyes, 2007).

La aplicación total consistió en: 10 talleres de 2 horas cada uno (a excepción de los talleres 2 y 10) dirigidos a los estudiantes, quienes estaban divididos en tres aulas de clase (de 39, 38 y 38 niños respectivamente) para facilitar el manejo del grupo y la interacción entre ellos. Por lo tanto, el mismo taller fue repetido tres veces. Los contenidos de los talleres, enunciados en la tabla 1, se relacionan con las características asociadas a factores personales de la resiliencia encontrados de forma consistente en algunos estudios recientes de resiliencia en niños, y evaluados en el instrumento utilizado para esta investigación. Estos son: autoestima, empatía, autonomía, humor y creatividad (Acevedo y Mondragón, 2005; Casillas, 2008; Gaxiola, Frías et ál., 2011; González-Arratia, 2016; Lemos et ál., 2016; Palacios y Monroy, 2011; Salgado, 2005a; 2005b; Sánchez et ál., 2015). En el caso que se requiera, la investigadora principal estará disponible para ampliar la información del diseño del programa y los contenidos de los talleres a quien lo requiera, debido a la extensión de estos. 
Tabla 1. Contenidos de la intervención con estudiantes

\begin{tabular}{|c|c|}
\hline Taller & Contenido \\
\hline $\begin{array}{l}\text { 1. Introducción al programa } \\
\text { Duración: } 2 \mathrm{~h}\end{array}$ & $\begin{array}{l}\text { Construcción conjunta (participantes e investigadora) de acuerdos de convivencia } \\
\text { para el espacio y puesta en común de las generalidades del programa. Exploración } \\
\text { de expectativas e inquietudes. Lectura dialógica del libro de literatura infantil Ramón } \\
\text { Preocupón de Anthony Browne (2007), como el medio para la expresión de las } \\
\text { preocupaciones, temores y situaciones adversas. }\end{array}$ \\
\hline $\begin{array}{l}\text { 2. Construcción del símbolo } \\
\text { del programa } \\
\text { Duración: } 4 \text { sesiones de } 2 \mathrm{~h}\end{array}$ & $\begin{array}{l}\text { Establecimiento del rapport con los participantes y promoción de la capacidad creativa } \\
\text { por medio de elaboración de artesanías llamadas "muñequitos quitapesares", en } \\
\text { coherencia con la lectura realizada la sesión anterior. Los materiales son proporcionados } \\
\text { por los mismos estudiantes; ellos se los procuraron a través de familiares o comunidad } \\
\text { circundante. }\end{array}$ \\
\hline $\begin{array}{l}\text { 3. Reconstrucción de } \\
\text { aprendizajes } \\
\text { Duración: } 2 \text { h }\end{array}$ & $\begin{array}{l}\text { Reflexión para favorecer las acciones organizadas, intencionadas, propositivas y críticas } \\
\text { de los participantes y desarrollar su autonomía. Las actividades de esta sesión se focalizan } \\
\text { en una retroalimentación por parte de los estudiantes, con el objetivo de identificar los } \\
\text { aprendizajes clave, las prácticas que se deben fortalecer y analizar las dificultades para } \\
\text { establecer acciones de mejora. }\end{array}$ \\
\hline $\begin{array}{l}\text { 4. Reconocimiento de } \\
\text { emociones } \\
\text { Duración: } 2 \mathrm{~h}\end{array}$ & $\begin{array}{l}\text { Identificación de emociones básicas y su relación con el comportamiento, a partir de } \\
\text { la discusión de la película de animación Inside Out de Disney y Pixar (2015). Antes de } \\
\text { verla, se verifican los conocimientos previos de los participantes sobre el tema y se hace } \\
\text { una sensibilización para orientar la reflexión individual y grupal, como insumo para la } \\
\text { siguiente sesión. }\end{array}$ \\
\hline $\begin{array}{l}\text { 5. Expresión y gestión de las } \\
\text { emociones } \\
\text { Duración: } 2 \mathrm{~h}\end{array}$ & $\begin{array}{l}\text { Promoción de la comprensión emocional en sí mismos y en los demás para promover } \\
\text { autoestima y empatía. Cada participante dibuja la emoción que considera predominante } \\
\text { en él, siguiendo los modelos propuestos en la película. Luego, diligencian una ficha } \\
\text { didáctica donde deben identificar qué situación, objeto, lugar, etc. le genera determinada } \\
\text { emoción. }\end{array}$ \\
\hline $\begin{array}{l}\text { 6. Exploración de fortalezas } \\
\text { Duración: } 2 \mathrm{~h}\end{array}$ & $\begin{array}{l}\text { Acontecimientos personales positivos y negativos donde reconozcan fortalezas que } \\
\text { estimulen su autoestima. Esto utilizando un esquema denominado por los participantes } \\
\text { "El camino de la vida", donde ubican cinco momentos muy significativos que hayan } \\
\text { marcado su trayectoria de vida. No tienen que ser grandes acontecimientos, pero sí los } \\
\text { que recuerden de manera especial. }\end{array}$ \\
\hline $\begin{array}{l}\text { 7. Experiencias resilientes } \\
\text { Duración: } 2 \mathrm{~h}\end{array}$ & $\begin{array}{l}\text { Discusión de historias de vida que permitan promover el factor empatía y la resiliencia. } \\
\text { Se presentan algunas historias de vida de personas reales que han tenido que enfrentar y } \\
\text { superar situaciones adversas, resaltando cómo lograron resignificar el dolor y fortalecerse } \\
\text { por medio de esas experiencias. Aquí se solicita la participación de miembros de la } \\
\text { comunidad circundante. }\end{array}$ \\
\hline
\end{tabular}




\begin{tabular}{|c|c|}
\hline Taller & Contenido \\
\hline $\begin{array}{l}\text { 8. Disminución de tensiones } \\
\text { Duración: } 2 \text { h }\end{array}$ & $\begin{array}{l}\text { Vinculación libre de los participantes en actividades como coloreado de mandalas o yoga } \\
\text { de la risa para trabajar de forma autónoma, creativa y humoristica, la disminución de } \\
\text { tensiones, el estrés, y como alternativa de expresión cuando se sientan enojados o tristes. } \\
\text { Además, puesta en común de situaciones adversas que sean frecuentes entre ellos y } \\
\text { generen conflicto en espacios de interacción. }\end{array}$ \\
\hline $\begin{array}{l}\text { 9. Capacidad de soñar } \\
\text { Duración: } 2 \text { h }\end{array}$ & $\begin{array}{l}\text { Puesta en común de expectativas y sueños a futuro de los participantes, fomentando su } \\
\text { autoestima, autonomia y creatividad. Esta sesión se crea a partir del libro Willy el soñador } \\
\text { de Anthony Browne (2014). Se utiliza la proyección de un video que muestra la historia } \\
\text { a partir de las ilustraciones del libro mientras una voz la cuenta con sonidos y tonos } \\
\text { adecuados para fomentar su imaginación. }\end{array}$ \\
\hline $\begin{array}{l}\text { 10. Cierre del programa } \\
\text { Duración: } 3 \text { sesiones de } 2 \text { h }\end{array}$ & $\begin{array}{l}\text { Representación simbólica y artesanal de los aprendizajes generados a partir del programa } \\
\text { y de las proyecciones a futuro de cada participante. Se retoma el tema de expectativas y } \\
\text { sueños iniciado en la sesión anterior, y a partir de la elaboración de un "Portarretrato de } \\
\text { los sueños" que se convierte en la ventana hacia el futuro, se visualizan metas y objetivos. }\end{array}$ \\
\hline
\end{tabular}

Fuente: Elaboración propia

En el caso de los padres y acudientes, se realizaron 4 talleres de 2 horas cada uno bajo la dinámica de escuela de padres, sus contenidos se refieren en la tabla 2. Estos no fueron diseñados por las investigadoras, pero sí ajustados a la propuesta del programa de intervención y a las necesidades de los participantes.
Hacen parte de la publicación Tejiendo vínculos, tejiendo sueños, tejiendo vida desde la primera infancia. Cartilla para la promoción de la resiliencia familiar (Ministerio de Educación Nacional (MEN); Instituto Colombiano de Bienestar Familiar (ICBF) y Organización Internacional para las Migraciones, (oIM), 2008).

Tabla 2. Contenidos de la intervención con padres de familia o acudientes

\begin{tabular}{|l|l|}
\multicolumn{1}{|c|}{ Taller } & \multicolumn{1}{c|}{ Contenido } \\
\hline $\begin{array}{l}\text { 1. Introducción al programa } \\
\text { Duración: } 2 \mathrm{~h}\end{array}$ & $\begin{array}{l}\text { Explicación de generalidades del programa y presentación de la cartilla que orienta las } \\
\text { sesiones posteriores. }\end{array}$ \\
\hline $\begin{array}{l}\text { 2. Reconocimiento de vínculos } \\
\text { Duración: } 2 \mathrm{~h}\end{array}$ & $\begin{array}{l}\text { Reflexión sobre la importancia de los vínculos afectivos que se construyen desde la } \\
\text { concepción y sobre los acontecimientos significativos en la infancia. }\end{array}$ \\
\hline $\begin{array}{l}\text { 3. Emociones y crianza } \\
\text { Duración: } 2 \mathrm{~h}\end{array}$ & $\begin{array}{l}\text { Identificación de emociones en el cuerpo y relación con las prácticas de crianza que ellos } \\
\text { recibieron frente a la que están ofreciendo actualmente a sus hijos. }\end{array}$ \\
\hline $\begin{array}{l}\text { 4. Futuros posibles y deseables } \\
\text { Duración: } 2 \mathrm{~h}\end{array}$ & $\begin{array}{l}\text { Reconocimiento de sueños a nivel individual y familiar, estableciendo estrategias y } \\
\text { procesos para lograrlos y ser inspiración para sus hijos. }\end{array}$ \\
\hline
\end{tabular}


Con las tres docentes directoras de curso se realizó una evaluación de los factores de riesgo y de los factores protectores presentes en cada estudiante, clasificados en los sistemas propuestos en el modelo ecológico de Bronfenbrenner (1993/1994) y Belsky (1980). Esto por medio de un instrumento de informe diseñado por las investigadoras para establecer el nivel de riesgo y de protección en el que se encontraba cada niño. Se utilizaron para tal fin 13 horas de trabajo pedagógico individual, con cada una, establecidas en su horario laboral.

Respecto a la comunidad circundante, el programa promovió que los estudiantes estuvieran adscritos a actividades extraescolares, 4 horas semanales, dirigidas por entidades en convenio con la escuela y desarrolladas en escenarios públicos de la localidad. Cada estudiante podia elegir una actividad deportiva y una artística de acuerdo con su interés, y participar en ella al finalizar la jornada escolar regular.

Postest:

Una vez finalizado el programa, se llevó a cabo la aplicación del postest (noviembre de 2017) siguiendo los mismos criterios que en el pretest. No se presentó ningún tipo de inconveniente en su resolución y fue realizado por la totalidad de la muestra.

\section{Consideraciones éticas}

De acuerdo con los principios establecidos en el Manual de Publicaciones de la American Psychological Association (APA, 2010), la Declaración de Helsinki de la Asociación Médica Mundial (AMM, 2017), el Informe Belmont (1979) y la Resolución 8430 (1993) para Colombia, este estudio se realizó conforme a los criterios éticos expuestos en la normatividad internacional y nacional mencionada. Se contó con el asentimiento informado y por escrito de los participantes menores de edad, y el consentimiento informado y por escrito de sus representantes legales mayores de edad.

\section{Análisis estadístico}

El análisis estadístico de los resultados se efectuó por medio del programa Statistical Package for Social Sciences (spss) versión 24.0. El nivel de significación empleado fue de $p=, 05$.
Se realizó el análisis de normalidad de Kolmogorov-Smirnov para identificar si los datos se asemejaban a la distribución normal. Se obtuvieron los estadísticos descriptivos y frecuencias de los puntajes alcanzados por los participantes en cada factor personal resiliente y de los puntajes generales de resiliencia, tanto en el pretest como en el postest. Para comparar los datos obtenidos de las dos muestras relacionadas (pretest y postest) se ejecutó la prueba de Wilcoxon. El efecto del programa se evaluó mediante diferencia de medias.

\section{Resultados}

En la tabla 3 se muestra la prueba de bondad de ajuste a la curva normal de Kolmogorov-Smirnov, puesto que la muestra es mayor a 50 participantes. De acuerdo con estos resultados, se observa que los puntajes alcanzados en los 5 factores personales de la resiliencia (autoestima, empatía, autonomía, humor y creatividad), tanto en la medición pretest como en la postest, arrojan índices significativos de $p=, 000$ $(p .<, 05)$. En resiliencia general, teniendo en cuenta que es la sumatoria de los 5 factores personales, se observan índices significativos de $p=, 067$ en el pretest, y $p=, 200^{*}$ en el postest. Como estos últimos datos son los únicos que se asemejan a una distribución normal, en las pruebas subsecuentes se utiliza estadistica no paramétrica.

Tabla 3. Análisis de normalidad

\begin{tabular}{|c|c|c|c|c|c|c|}
\hline \multicolumn{7}{|c|}{ Kolmogorov-Smirnov } \\
\hline & \multicolumn{3}{|c|}{ Pretest } & \multicolumn{3}{|c|}{ Postest } \\
\hline & 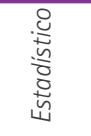 & $g l$ & Sig. & 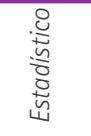 & gl & Sig. \\
\hline Autoestima &, 145 & 115 &, 000 & ,200 & 115 &, 000 \\
\hline Empatía & , 175 & 115 &, 000 & ,139 & 115 &, 000 \\
\hline Autonomía &, 178 & 115 &, 000 & , 161 & 115 & ,000 \\
\hline Humor &, 166 & 115 &, 000 &, 144 & 115 &, 000 \\
\hline Creatividad &, 177 & 115 &, 000 & , 180 & 115 &, 000 \\
\hline $\begin{array}{l}\text { Resiliencia } \\
\text { general }\end{array}$ & ,080 & 115 &, 067 &, $200^{*}$ & 115 &, 000 \\
\hline
\end{tabular}

*Est es un limite inferior de la significación verdadera. Fuente: Elaboración propia 
En la tabla 4 se presentan los principales estadisticos descriptivos obtenidos en el pretest y el postest. Se evidencia que las medias aumentan en los 5 factores resilientes y en resiliencia general, comparando el puntaje de la evaluación pre y la evaluación post. Los demás estadísticos también presentan variaciones en su mayoría.

Tabla 4. Estadísticos descriptivos de los factores personales de resiliencia y de resiliencia general en el pretest y el postest

\begin{tabular}{|c|c|c|c|c|c|c|c|}
\hline Autoestima (pretest) & 7,37 & 8 & 7,00 & 2,113 & 1,454 & 3 & 10 \\
\hline Autoestima (postest) & 7,98 & 9 & 8,00 & 2,649 & 1,628 & 4 & 10 \\
\hline Empatia pretest) & 6,70 & 6 & 7,00 & 1,877 & 1,370 & 4 & 9 \\
\hline mánáximo
\end{tabular}

Fuente: Elaboración propia

Para establecer si estas diferencias en las medias son estadisticamente significativas, y si el programa de intervención escolar tuvo efecto en los factores resilientes y la resiliencia general de los participantes respecto de la primera medición con la segunda, se utiliza la prueba Wilcoxon (Véase tabla 5). Los resultados señalan un índice de significación asintótica unilateral mayor a,05 (p.>,05) en los factores empatía, autonomía y creatividad. Se rechaza la hipótesis de trabajo, se acepta la nula y se concluye que no existe diferencia estadisticamente significativa entre el pretest y el postest de los factores mencionados, por lo tanto, el programa de intervención no aumentó los niveles de empatía, autonomía y creatividad de los participantes.

Por su parte, los factores autoestima y humor presentan un índice de significación asintótica unilateral menor a ,05 (p.<,05), lo que permite aceptar la hipótesis de trabajo, rechazar la nula y concluir que existe diferencia estadisticamente significativa entre la evaluación pre y post. En este caso, el programa aumentó los niveles de autoestima y humor 
de los participantes. Lo mismo sucede con resiliencia general dada su significación asintótica unilateral de $p=, 012^{*}(p .<, 05)$. Este resultado sugiere que los niños en condición de vulnerabilidad social que participaron en el programa de intervención escolar Tejedores de Resiliencia alcanzan mayores niveles de resiliencia general, aceptándose la hipótesis de trabajo y rechazándose la nula.

Tabla 5. Prueba de rangos con signo de Wilcoxon

\begin{tabular}{|c|c|c|c|c|c|c|}
\hline & \multicolumn{5}{|c|}{ Factores personales de resiliencia } & \multirow{2}{*}{$\begin{array}{c}\text { Resiliencia } \\
\text { General } \\
\text { (pretest- } \\
\text { postest) }\end{array}$} \\
\hline & $\begin{array}{c}\text { Autoestima } \\
\text { (pretest- } \\
\text { postest) }\end{array}$ & $\begin{array}{l}\text { Empatia } \\
\text { (pretest- } \\
\text { postest) }\end{array}$ & $\begin{array}{c}\text { Autonomía } \\
\text { (pretest- } \\
\text { postest) }\end{array}$ & $\begin{array}{l}\text { Humor } \\
\text { (pretest- } \\
\text { postest) }\end{array}$ & $\begin{array}{c}\text { Creatividad } \\
\text { (pretest- } \\
\text { postest) }\end{array}$ & \\
\hline Z & $-3,362^{\mathrm{a}}$ & $-1,099^{a}$ & $-1,552^{\mathrm{a}}$ & $-1,751^{b}$ & $-1,437^{a}$ & $-2,236^{a}$ \\
\hline Sig. asintótica &, $000^{*}$ &, 136 &, 060 &, $04^{*}$ &, 075 &, $012^{*}$ \\
\hline
\end{tabular}

a Se basa en rangos positivos - ${ }^{b}$ Se basa en rangos negativos - *Estadisticamente significativo

\section{Discusión}

El objetivo de esta investigación fue evaluar la efectividad del programa de intervención escolar Tejedores de Resiliencia sobre los niveles de resiliencia de un grupo de niños expuestos a condiciones de vulnerabilidad social. Los resultados mostraron que, los niveles de resiliencia general aumentaron de modo significativo en los participantes, aceptándose la hipótesis de trabajo. En un análisis por factores, la intervención afectó básicamente la autoestima y el humor. Respecto a la empatía, la autonomía y la creatividad el aumento no fue significativo. Podría sugerirse entonces que la intervención fue efectiva y se alcanzó el objetivo propuesto.

Estos resultados son coherentes con los presentados en la evaluación de la efectividad de otros programas. Reyes (2007), quien utilizó el mismo instrumento de medida empleado para este estudio, mostró un aumento en los niveles de resiliencia general luego de su intervención, tal como sucedió en esta experiencia. En relación con el análisis por factores la autoestima, la autonomía, el humor y la creatividad no presentaron cambios significativos. No obstante, la empatía sí tuvo un efecto positivo. En el caso de Tejedores de Resiliencia, la implementación del programa aumenta la autoestima y el humor, lo cual implica movimientos en más factores de los que referencia el autor. Este hallazgo permite observar que el tipo de intervención parece estar asociado a la manera como los factores de la resiliencia se modifican y, por ende, la revisión de las estructuras para cada programa es fundamental para mejorar las afectaciones.

En cuanto a la autoestima es ampliamente relacionada con la resiliencia y se considera un factor predictor central de esta (González-Arratia, 2016; Palacios y Monroy, 2011; Salgado, 2005a; 2005b). El programa llevado a cabo por Lynch et ál. (2004) abordó la construcción de autoestima como un dominio conceptual clave para desarrollar resiliencia y obtuvo resultados significativos después de su implementación. La intervención propuesta por Coholic et ál. (2012) buscó ante todo mejorar la autoestima de los participantes y al mismo tiempo aumentar sus niveles de resiliencia, propósito que alcanzó de acuerdo con los resultados reportados. En Tejedores de Resiliencia, las actividades encaminadas al fortalecimiento de la autoestima de los niños ocuparon un lugar central en el proceso, puesto que se estimó que poseer una valoración positiva de sí mismos les permitiría confiar en sus recursos internos y utilizarlos para enfrentar la adversidad. Esta podría ser la razón del resultado significativo de dicho factor entre las dos 
mediciones. En este sentido, la pregunta estaría dirigida a responder si solo afectando la autoestima se consiguen cambios en los niveles generales de resiliencia, sin embargo, no se conocen investigaciones que se hayan propuesto resolverla.

El factor humor es una forma de autoprotección, un mecanismo para afrontar los problemas cotidianos y las dificultades situacionales. Se asocia directamente con la resiliencia en tanto que permite relativizar y positivar los problemas (Gaxiola et ál., 2011; Omar et ál, 2011; Palacios y Monroy, 2011; Salgado, 2005a; 2005b). A pesar de nombrarse con frecuencia en la literatura sobre resiliencia, son escasos los estudios empíricos que amplian la información sobre este factor, como tampoco se muestra claramente cómo desarrollarlo en las personas. No obstante, se mencionan juegos y actividades para practicar en el ámbito familiar y educativo que ayudan a estimularlo (Fiorentino, 2008). En Tejedores de Resiliencia no se hicieron actividades que tuvieran como objetivo directo aumentar el humor de los participantes, pero la preocupación de la investigadora principal por mantener un clima de intervención positivo que asegurara la permanencia de los estudiantes, su interacción, y asimismo afectara positivamente la retención de los aprendizajes, favoreció su aparición como factor constitutivo en los espacios de encuentro y registró un cambio significativo.

Los factores empatía, autonomía y creatividad, como ya se mencionó, se encuentran de modo consistente en algunos estudios recientes de resiliencia en niños (Acevedo y Mondragón, 2005; Casillas, 2008; Gaxiola et ál., 2011; Lemos et ál., 2016; Salgado, 2005a; 2005b; Sánchez et al., 2015). Sin embargo, no proponen acciones directas de intervención. Tejedores de Resiliencia pretendió afectarlos en los diferentes talleres realizados, pero no fueron trabajados con el mismo énfasis y transversalidad que el factor autoestima y tampoco hicieron parte de la constitución de los espacios de encuentro como el factor humor. Esta inferencia podría explicar los resultados en los cinco factores de acuerdo con la comparación de medias en las dos mediciones.

En cuanto a la participación de los padres de familia o acudientes de los estudiantes en el programa es posible señalar que fue beneficiosa para alcanzar el objetivo de la intervención. Nettles et ál. (2000), quienes enfatizan la importancia de los recursos sociales en el desarrollo de la resiliencia, afirman que aquellas estrategias que incorporan también a los padres o acudientes permiten predecir un mejor resultado en los programas de intervención. Asimismo, la participación en actividades extracurriculares y las relaciones de apoyo con los docentes tienen efectos positivos. Toda la información recolectada en los diferentes talleres y actividades implementadas en Tejedores de Resiliencia puede ser analizada en próximas investigaciones, de manera que sea posible obtener datos concluyentes acerca del papel que juegan dichos acompañamientos y agentes en la mejoría de los niveles de resiliencia de los estudiantes. Sin embargo, en la medida en que no era el objetivo central de esta investigación, dichos datos no se exploraron en profundidad.

El estudio realizado presenta limitaciones que es importante señalar, en especial en el método. Dado que no contó con un grupo control, se hace difícil atribuir al programa el aumento de los niveles de resiliencia de los niños y no es posible descartar que los cambios encontrados se deban a otras variables diferentes de la intervención realizada. No obstante, fue decisión de las investigadoras ser equitativas en la participación del grupo de estudiantes en la intervención, respetar sus tiempos y espacios, y vincular Tejedores de Resiliencia a las actividades cotidianas de la escuela, lo cual le hace ganar en validez ecológica. Será un desafio metodológico y ético de futuras evaluaciones, por tanto, realizar intervenciones con mayor control en este tipo de programas. Además, los resultados de este trabajo se ajustan a una muestra poblacional seleccionada intencionalmente, por lo tanto, para lograr una mayor generalización, es importante replicarlo con muestras mayores de niños en condición de vulnerabilidad social de otras edades y niveles educativos.

Pese a estas limitaciones, el estudio entrega información valiosa sobre la intervención en resiliencia con niños expuestos a condiciones de vulnerabilidad social. Investigaciones de esta naturaleza contribuyen a la implementación de estrategias efectivas que incrementen en ellos habilidades personales, conductas 
dirigidas a la superación y el éxito a pesar de la adversidad, y la capacidad de reconocer oportunidades para fortalecerse y ser transformados positivamente aún en contextos desfavorables. De igual manera, se realizó en la escuela, con la participación de padres o acudientes, docentes y comunidad circundante, en este sentido adquiere un valor adicional como método educativo innovador dado que muestra la posibilidad de entrar a las aulas para trabajar desde allí en el mejoramiento de las condiciones de vida de los estudiantes y renovar el sentido mismo de los actos educativos. Sin lugar a dudas, requiere de mayor compromiso por parte de todos los agentes vinculados al ámbito escolar, pero resulta un reto interesante para comprender y mejorar las situaciones de adversidad y estrés significativo que vive cada estudiante.

Investigaciones ulteriores pueden explorar, como se mencionó antes, si los 5 factores resilientes deben ser tenidos en cuenta para una intervención escolar y si todos deben trabajarse con la misma intensidad; si la estructura interna de los programas puede presentarlos de manera escalonada (uno después del otro) o continua (simultáneamente); y si su peso ponderado implica cambios significativos en cada uno de los cinco factores estudiados. Del mismo modo, futuras evaluaciones deben incorporar otras mediciones complementarias de resultados o considerar evaluaciones de jueces externos a la intervención antes de iniciar la implementación del programa.

\section{Referencias}

American Psychological Association. (2010). Manual de Publicaciones de la American Psychological Association. El Manual Moderno.

Asociación Médica Mundial. (2017). Declaración de Helsinki de la Amm-Principios éticos para las investigaciones médicas en seres humanos. https://www.wma.net/es/ policies-post/declaracion-de-helsinki-de-la-amm-principios-eticos-para-las-investigaciones-medicas-en-seres-humanos/

Acevedo, V. y Mondragón, H. (2005). Resiliencia y escuela. Pensamiento Psicológico, 1(5), 21-35.

Becoña, E. (2006). Resiliencia: definición, características y utilidad del concepto. Revista de Psicopatología y Psicología Clínica, 11(3), 125-146.
Belsky, J. (1980). Child maltreatment: an ecological integration. American Psychologist Association, 35(4), 320-335. doi: 10.1037/0003-066X.35.4.320

Bronfenbrenner, U. (1994). Ecological models of human development. En International Encyclopedia of Education. Vol. 3. Elsevier.

Cabrera, V. E., Aya, V. L. y Guevara, I. P. (2014). Diseño y análisis psicométrico de una escala de resiliencia en infantes. Revista de la Facultad de Medicina, 62(2), 213-220.

Campo, R., Granados, L. F., S.J., Muñoz, L., Rodríguez, M. S. \& Trujillo, S. (2012). Caracterización del avance teórico, investigativo y/o de intervención en resiliencia desde el ámbito de las universidades en Colombia. Universitas Psychologica, 11(2), 545-557

Casillas, J. (2008). ¿Por qué Miriam sí va a la escuela? Resiliencia en la educación básica mexicana. Revista Mexicana de Investigación Educativa, 13(39), 1255-1279.

Coholic, D., Eys, M. y Lougheed, S. (2012). Investigating the effectiveness of an arts-based and mindfulness-based group program for the improvement of resilience in children in need. Journal of Child and Family Studies, 21, 883-844. https://doi.org/10.1007/s10826-011-9544-2

Comisión Nacional para la protección de los sujetos humanos de investigación biomédica y comportamental. (1979). Informe Belmont. http://www.bioeticayderecho.ub.edu/ archivos/norm/InformeBelmont.pdf

Fiorentino, M. (2008). La construcción de la resiliencia en el mejoramiento de la calidad de vida y la salud. Suma Psicológica, 15(1), 95-113.

Gaxiola, J., Frías, M., Hurtado, M., Salcido, L. y Figueroa, M. (2011). Validación del inventario de resiliencia (ires) en una muestra del noroeste de México. Enseñanza e Investigación en Psicología, 16(1), 73-83.

González-Arratia, N. I. (2016). Autoestima y apoyo social sobre la resiliencia en niños con y sin depresión. Revista Iberoamericana de Producción Académica y Gestión Educativa, 3(6). http://www.pag.org.mx/index.php/PAG/ article/view/603

González-Arratia, N. I. y Valdez, J. (2013). Resiliencia: Diferencias por edad en hombres y mujeres mexicanos. Acta de investigación psicológica, 3(1), 941-955.

Lázaro, S. (2009). Resiliencia en niños y adolescentes: revisión teórica e implicaciones para la intervención psicoeducativa en situaciones de maltrato familiar. Estudios de Psicología: Studies in Psychology, 30(1), 89-104.

Lemos, V., Krum, G., Gutiérrez M. y Arán-Filippetti, V. (2016). Desarrollo de una escala para evaluar recursos de personalidad asociados a la resiliencia infantil. Acción Psicológica, 13(2), 101-116. https://doi.org/10.5944/ ap.13.2.17821 
Luthar, S. S., Cicchetti, D. y Becker, B. (2000). The construct of resilience: A critical evaluation andguidelines for future work. Child Development, 71(3), 543-562. http://dx.doi. org/10.1111/1467-8624.00164.

Lynch, K., Geller, S., \&t Schmidt, M. (2004). Multi-year evaluation of the effectiveness of a resilience-based prevention program for young children. Journal of Primary Prevention, 24(3), 335-353.

Masten, A. (2001). Ordinary magic: resilience processes in development. American Psychologist Association, 56(3), 227-238

Ministerio de Educación Nacional, Instituto Colombiano de Bienestar Familiar y Organización Internacional para las Migraciones. (2008). Tejiendo vínculos, tejiendo sueños, tejiendo vida desde la primera infancia. Cartilla para la promoción de la resiliencia familiar. https://web.oas.org/ childhood/ES/Lists/Recursos\%20\%20Bibliografia/Attachments/61/66.pdf

Morelato, G. (2011). Resiliencia en el maltrato infantil: aportes para la comprensión de factores desde un modelo ecológico. Revista de Psicología, 29(2), 203-224.

Nettles, S., Mucherah, W. y Jones, D. (2000). Understanding resilience: the role of social resources. Journal of Education for Students Placed at Risk, 5(1, 2), 47-60.

Obando, O., Villalobos, M. y Arango, S. (2015). Resiliencia en niños con experiencias de abandono. Acta Colombiana de Psicología, 13(2), 149-159.

Omar, A., Paris, L., Uribe, H., Ameida da Silva Junior, S. y Aguiar de Souza, M. (2011). Un modelo explicativo de resiliencia en jóvenes y adolescentes. Psicología em Estudo, 16(2), 269-277.

Palacios, L. y Monroy, B. G. (2011). Resiliencia: ¿Es posible medirla e influir en ella? Salud Mental, 34(3), 237-246.

Perona, N. y Rocchi, G. (2001). Vulnerabilidad y exclusión social. Una propuesta metodológica para el estudio de las condiciones de vida de los hogares. Revista Kairos, 8. https://www.revistakairos.org/vulnerabilidad-y-exclusion-social-una-propuesta-metodologica-para-el-estudio-de-las-condiciones-de-vida-de-los-hogares/

Resolución 8430 de 1993. Normas científicas, técnicas y administrativas para la investigación en salud (04 oct, 1993). Ministerio de Salud de la República de Colombia.
Reyes, M. (2007). Efectos del programa "descubriendo la vida" sobre los niveles bajos de resiliencia en un grupo de estudiantes. Revista Académica, 33, 61-75.

Richaud, M. C. (2013). Contributions to the study and promotion of resilience in socially vulnerable children. American Psychologist Association, 68(8), 751-758. https:// psycnet.apa.org/doi/10.1037/a0034327

Rodríguez, H., Guzmán, L. y Yela, N. (2012). Factores personales que influyen en el desarrollo de la resiliencia en niños y niñas en edades comprendidas entre 7 y 12 años que se desarrollan en extrema pobreza. International Journal of Psychological Research, 5(2), 98-107.

Ruiz, N. (2012). La definición y medición de la vulnerabilidad social. Un enfoque normativo. Investigaciones Geográficas $(M x), 77,63-74$.

Rutter, M. (2012). Resilience as a dynamic concept. Development and psychopathology. 24, 335-44. https://doi. org/10.1017/S0954579412000028

Salgado, A. C. (2005a). Inventario de resiliencia para niños: fundamentación teórica y construcción. Cuaderno de Investigación No. 8. Instituto de Investigación. Escuela Profesional de Psicología. Universidad de San Martín de Porres.

Salgado, A. C. (2005b). Métodos e instrumentos para medir la resiliencia: una alternativa peruana. Liberabit. Revista de Psicología, 11(11), 41-48.

Sánchez, Ó., Méndez, F. y Garber, J. (2015). Producción divergente explicativa: la relación entre resiliencia y creatividad. Electronic Journal of Research in Educational Psychology, 13(3), 551-568.

Villalta, M. A. y Saavedra, E. (2012). Cultura escolar, prácticas de enseñanza y resiliencia en alumnos y profesores de contextos sociales vulnerables. Universitas Psychologi$\mathrm{ca}, 11(1), 67-78$.

Windle, G., Bennett, K. M. y Noyes, J. (2011). A methodological review of resilience measurement scales. Health and Quality of Life Outcomes, 9(8). http://dx.doi. org/10.1186/1477-7525-9-8 


\section{Diálogo del conocimiento}

En el programa piloto de intervención escolar "Tejedores de Resiliencia", se presenta un estudio que abre caminos para pensar en el diseño, desarrollo y ejecución de una puesta en marcha para favorecer la capacidad de resiliencia en niños y niñas, adicionalmente a comprender la influencia activa de varios factores y las interacciones que tiene el ser humano y su medio ambiente, que muy bien lo exponen las autoras al referirse al modelo presentado por Bronfenbrenner.

Colombia es un pais con adversidades y situaciones complejas en el ámbito de la paz. Históricamente, ha sufrido por varias décadas procesos de violencia, fenómeno complejo y multicausal que impacta a la familia y a sus generaciones, produciendo desarraigo, indiferencia y desesperanza, entre otras razones. Se hace un llamado inminente para las facultades, instituciones y comunidades que incidan en la formación de maestros y maestras, pues son ellos los agentes educativos para establecer espacios de dialogo, discusión, reflexión y diseño de propuestas que generen estrategias pedagógicas, como es el caso de las actividades rectoras. El arte, el juego, la exploración del medio y la literatura promueven un acompañamiento cercano, consiente engalanado por el afecto y buen trato para resignificar las vivencias propias y potencian los procesos de resiliencia en la vida de los niños y las niñas y sus familias.

Finalmente, este estudio permite reconocer que tanto la familia, la escuela y la sociedad tienen una capacidad enorme para generar mejores condiciones de vida para los niños y niñas. Cabe resaltar la gran influencia que permea a la educación infantil el contacto con sus docentes y acciones pedagógicas diseñadas con intencionalidades claras y argumentativas que contribuyan al desarrollo integral de los infantes. Esto, sin lugar a dudas, inunda de sentido el quehacer del maestro y la maestra en la implementación de propuestas significativas que se convierten en un 


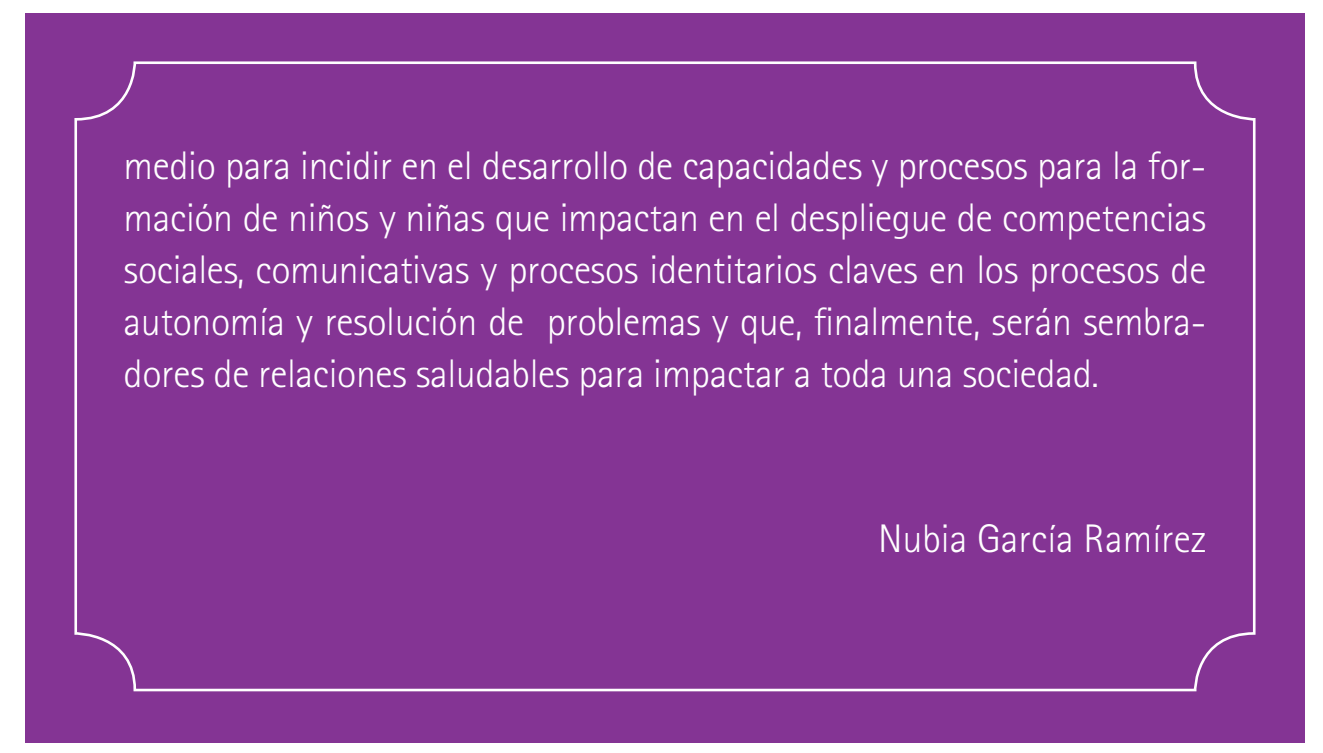

